\title{
Los grupos interactivos en Educación Física: Una experiencia docente en Educación Secundaria Interactive Groups in Physical Education: A Teaching Experience in Secondary Education Javier Olaya Cuartero, Miguel García Jaén, Salvador García Martínez, Alberto Ferriz Valero Universidad de Alicante (España)
}

Resumen. El proyecto de investigación de más rango científico y con los mayores recursos dedicados al estudio de la educación escolar en el Programa Marco de la Unión Europea: INCLUD-ED, muestra que las prácticas de las escuelas de éxito en Europa están en la línea del enfoque dialógico del aprendizaje. En un centro educativo donde el alumnado pertenece a 36 nacionalidades diferentes, se propone la implantación de formas dialógicas de organización en el aula como los grupos interactivos. El objetivo del presente estudio fue analizar el tipo de agrupación en el aula mediante grupos interactivos para comprobar, por un lado, su impacto sobre la motivación y la satisfacción de las necesidades psicológicas básicas en el ejercicio, y por otro, en qué medida influyen estos grupos interactivos sobre el aprendizaje teórico. Para esto, 113 participantes en la asignatura de Educación Física de tercero y cuarto de Educación Secundaria Obligatoria recibieron dos clases mediante organizaciones diferentes del aula, una organización tradicional (TRAD) a modo de grupo de control y otra de grupos interactivos (GIN) respectivamente. Se evaluó el grado de satisfacción de las necesidades psicológicas básicas en el ejercicio, el grado de motivación y la adquisición de conocimientos teóricos específicos de la asignatura. Los resultados muestran tanto mejoras a nivel de conocimientos teóricos, como una mayor satisfacción de las necesidades psicológicas básicas en el ejercicio y motivación intrínseca de GIN frente a TRAD.

Palabras clave. Agrupación del Aula, Comunidades de Aprendizaje, Alumnado, Necesidades Psicológicas Básicas en el Ejercicio, Motivación.

\begin{abstract}
The research project with the highest scientific rank and the greatest resources dedicated to the study of school education in the European Union's Framework Programme: INCLUD-ED, shows that the practices of successful schools in Europe are in line with the dialogical approach to learning. In an educational school where students belong to 36 different nationalities, the implementation of dialogical forms of organization in the classroom, such as interactive groups, is proposed. The present study aimed to analyze the type of grouping in the classroom through interactive groups in order to check, on the one hand, their impact on motivation and the satisfaction of basic psychological needs, and on the other hand, to what extent these interactive groups influence theoretical learning. For this purpose, 113 participants in the subject of Physical Education, from third and fourth year of Compulsory Secondary Education, received two classes through different classroom organizations: a traditional organization (TRAD) as a control group, and with interactive groups (GIN), respectively. The degree of satisfaction of basic psychological needs, the degree of motivation, and the acquisition of specific theoretical knowledge of the subject were evaluated. The results show both improvements in theoretical knowledge and greater satisfaction of basic psychological needs and intrinsic motivation of GIN compared to TRAD.
\end{abstract}

Keywords. Classroom Grouping, Learning Community, Students, Basic Psychological Needs in Exercise, Motivation.

\section{Introducción}

En los últimos años, con la aparición de nuevas ideas en Educación Física (EF), han emergido nuevos métodos de educación (Zhang, 2016). Los estilos de enseñanza cognoscitivos se diferencian de los tradicionales en el empleo de la indagación y la búsqueda (Muros, Som, Leyva, \& Zabala, 2010). Los planteamientos metodológicos tradicionales, se relacionan con un clima de aula orientado al ego, mientras que los planteamientos basados en un estilo actitudinal lo hacen con un clima más orientado a la tarea (Hortigüela-Alcalá, Fernández-Río, \& Pérez-Pueyo, 2016). En esta línea, destaca por su importancia el trabajo a través de metodologías activas que permiten el aumento del tiempo de compromiso motor en alumnado de Educación Secundaria en un área de carácter eminentemente práctico como la EF (García, Lemus, \& Morales, 2015). Ejemplo de ellos es el surgimiento de alternativas metodológicas como el aula invertida o Flipped Classroom que transforman las metodologías tradicionales vinculadas exclusivamente al aula, que focalizaban la atención en actividades de explicación, transmisión de contenidos y resolución de problemas (Ferriz, Sebastiá, \& García, 2017). También destaca la aparición de la

Fecha recepción: 10-08-19. Fecha de aceptación: 09-02-20

Alberto Ferriz Valero

alberto.ferriz@ua.es gamificación como una estrategia de aprendizaje emergente para la educación actual por llevar implícitas características positivas que favorecen la motivación e interés del alumnado por aprender, la mejora del rendimiento y un aumento del compromiso por la práctica de la actividad física (Escaravajal \& Martín-Acosta, 2019). Sin embargo, la creciente investigación en EF en las dos últimas décadas se ha focalizado en la atención en la importancia, eficacia y oportunidad asociadas a las Comunidades de Aprendizaje (CA) (Bolam, 2000; Desimone 2011) y a las Comunidades de Práctica (Deglau \& O'Sullivan, 2006; Patton, Parker, \& Pratt, 2013).

Con relación a las CA son definidas como un proyecto de éxito educativo que persigue una educación de calidad para todo el alumnado, al mismo tiempo que responden a las necesidades que plantea la sociedad actual y se oponen a las tendencias hacia la exclusión social que sufren los grupos más desfavorecidos, como pueden ser los inmigrantes o las minorías étnicas (Gómez, Mello, Santa Cruz, \& Sordé, 2010). También han sido descritas como grupos de personas que comparten una inquietud o pasión por algo que hacen y aprenden a hacerlo mejor mientras interactúan regularmente, buscando soluciones a los problemas y profundizando en los conocimientos y la experiencia mediante una interacción permanente (Wenger, 2013). Las CA surgen a partir de la noción de aprendizaje dialógico y de otras experiencias de éxito aplicadas en miles de escuelas de los Estados Unidos, 
llevándose a cabo en casi un centenar de centros en España y mostrando una gran eficacia en la tarea de acercar la escuela a la sociedad, en la superación del fracaso escolar y en la mejora de la convivencia (Capllonch \& Figueras, 2012). La transformación de la organización tradicional del centro a las CA, con el fin de optimizar los recursos para que todas las personas puedan acceder de forma fructífera a todas las posibilidades culturales y educativas, puede aportar para los alumnos desfavorecidos resultados educativos iguales o superiores a los de cualquier alumna o alumno que no forme parte de un grupo en riesgo de exclusión social (Elboj, Puigdellívol, Soler, \& Valls, 2002).

De este paradigma de CA, se desprenden los Grupos Interactivos (GIN). Los GIN, representan para Flecha (2001) el aspecto más radical del proyecto de CA, ya que no se saca a nadie del aula, pero una vez asumido que el profesorado no puede atender a todo el alumnado individualmente, se introducen en el aula todo tipo de recursos que son necesarios para acabar con los problemas de fracaso y conflicto. Estudios previos muestran que la implantación de formas dialógicas de organización en el aula como los GIN generan una mejora de los resultados académicos además de dinámicas solidarias entre el propio alumnado (Elboj \& Niemelä, 2010). La inclusión y el éxito educativo de los GIN en EF trasladan al aula los principios del aprendizaje dialógico, así lo hacen constar Castro, Gómez, y Macazaga (2014).

Para que sea posible un aprendizaje dialógico a través de GIN, el centro educativo deberá cumplir con siete principios necesarios: diálogo igualitario, inteligencia cultural, transformación, dimensión instrumental, creación de sentido, solidaridad e igualdad de diferencias (Aubert, García, \& Racionero, 2014). Esto es importante porque las escuelas de éxito en Europa están en la línea de este enfoque dialógico del aprendizaje, así lo muestra el proyecto de investigación de más rango científico y con los mayores recursos dedicados al estudio de la educación escolar en el Programa Marco de la Unión Europea: INCLUD-ED (Racionero \& Padrós, 2010). La importancia del aprendizaje dialógico ha sido previamente estudiada en centros educativos que optaron por la transformación en una CA para resolver un caso concreto, en el que una persona gitana respetada por la comunidad se ofrece para colaborar dentro del aula y resolver el comportamiento agresivo de uno de los alumnos gitanos con el profesor (Aubert, Flecha, García, Flecha, \& Racionero, 2008). Del mismo modo, también se han analizado los GIN en contextos similares, como organización del aula que podría resultar idónea para estudiar cómo la diversidad del voluntariado en las aulas puede promover la inclusión y el éxito de los niños de las minorías étnicas vulnerables (Valls \& Kyriakides, 2013).

Durante el proceso de enseñanza y aprendizaje llevado a cabo por el profesorado y alumnado también son importantes otros factores, entre ellos la satisfacción de las necesidades psicológicas básicas en el ejercicio y primarias y/o la motivación (Ruiz, Moreno, Ramón, \& Alias, 2015). El comportamiento humano es motivado por tres necesidades psicológicas básicas en el ejercicio y universales como son la autonomía (A), la competencia (C) y la relación con los demás (RD) (Deci \& Ryan, 1980). A su vez, estas necesidades influyen en la motivación y provocan una relación directa entre el aumento de éstas con la motivación intrínseca concretamente (Deci \& Ryan, 2000). Esto se refleja en una de las teorías motivacionales de mayor relevancia en la actualidad, la Teoría de la Autodeterminación o «self-determination theory» (Deci \& Ryan, 1985). Los tipos de motivación que recoge esta teoría pueden ordenarse desde los niveles más bajos hasta los niveles más altos en el siguiente orden: no motivación, regulación externa, regulación introyectada, regulación identificada y motivación intrínseca (Pelletier, Fortier, Vallerand, Tuson, Briere, \& Blais, 1995). Se complementa, a su vez con el Modelo Jerárquico de la Motivación Intrínseca y Extrínseca (Vallerand 1997, 2001). Este modelo diferencia tres niveles de análisis como el global, contextual y situacional que representan unos factores sociales, y darán lugar o no a la satisfacción de las necesidades psicológicas básicas en el ejercicio, provocando un nivel diferente de motivación (contextual y situacional) y dando lugar a una consecuencia diferente (afecto, cognición, y comportamiento) (Moreno, Zomeño, Marín, Cervelló, \& Ruiz, 2009).

En el contexto de la presente investigación, las CA cobran especial relevancia debido a que el centro educativo en el que se desarrolla sigue un proceso de transformación sociocultural que utiliza el aprendizaje dialógico basado en la educación participativa de la comunidad. Se trata de un centro de educación secundaria obligatoria (ESO) donde el alumnado pertenece a 36 nacionalidades diferentes, compuesto por una gran riqueza multicultural y multiétnica. Se ubica un barrio de renta media-baja y con una tasa elevada de absentismo, fracaso y abandono escolar, donde es de vital importancia conseguir un entorno desintoxicado para poder establecer un proceso correcto de enseñanza-aprendizaje. Surge la necesidad de estudiar la satisfacción de las necesidades psicológicas básicas en el ejercicio y motivación también en el contexto en el que se desarrolla la asignatura de EF, sin olvidar la adquisición de conocimientos teóricos específicos. Por tanto, el objetivo del presente estudio es comprobar si el empleo de la agrupación del aula con GIN tiene un impacto sobre la satisfacción de las necesidades psicológicas básicas en el ejercicio y de la motivación diferente a agrupaciones de aula de tipo tradicional (TRAD). En base a la literatura estudiada, la hipótesis previa plantea que el alumnado que recibe la clase mediante la agrupación de GIN aumentará tanto la satisfacción de las necesidades psicológicas básicas en el ejercicio y motivación intrínseca, como la adquisición de contenidos teóricos específicos de la asignatura, respecto al grupo que recibe la clase magistral mediante la organización tradicional.

\section{Material y método}

\section{Participantes}

Participaron 113 alumnas y alumnos ( $15.3 \pm 0.97$ años $)$ de tercero y cuarto de Educación Secundaria Obligatoria (ESO) de un centro educativo ubicado en la localidad de Alicante. Con previo acuerdo del tutor, se respetaron los grupos naturales con el objetivo de no romper la dinámica normal de la clase, con lo que fue aplicado un muestreo por conglomerados no probabilístico. Un grupo de control formado por 19 chicos y 31 chicas que recibieron los contenidos didácticos mediante una organización tradicional -TRAD- basada en una modificación del mando directo, mientras un segundo 
grupo lo recibió mediante la agrupación en el aula de grupos interactivos -GIN-, quedando compuesto por 33 chicos y 33 chicas. Previamente, todos los participantes entregaron el consentimiento informado de participación voluntaria en el estudio firmado por el padre, madre o tutor. Tras comenzar con una muestra inicial de 125 participantes, fueron excluidos del estudio 12 participantes que cumplieron algún criterio de exclusión: (1) no cumplimentar alguno de los cuestionarios o dejar algún ítem sin contestar y (2) no asistir presencialmente a las clases en las que se llevó a cabo la intervención.

\section{Procedimiento}

Se evaluaron la satisfacción de las necesidades psicológicas básicas en el ejercicio, motivación, así como la adquisición de conocimientos teóricos específicos de la asignatura relacionados con una unidad didáctica sobre la historia del fútbol, la técnica y táctica, las acciones y sistemas de juego $\mathrm{y}$ las posiciones en el campo.

El programa constó de 2 intervenciones por clase, para lo que fue necesario emplear 8 días de intervención, 4 para la primera semana y 4 para la segunda semana, que transcurrieron en el periodo temporal de un mes correspondiente al curso académico 2016/2017 (Tabla 1).

\begin{tabular}{|c|c|c|c|c|c|c|c|c|}
\hline \multirow{2}{*}{ Sesiones del } & \multicolumn{4}{|c|}{ Semana 1} & \multicolumn{4}{|c|}{ Semana 2} \\
\hline & Día 1 & Día 2 & Día 3 & Día 4 & Día 5 & Día 6 & Día 7 & Día 8 \\
\hline & \multicolumn{4}{|c|}{ Intervención } & \multirow{2}{*}{\multicolumn{4}{|c|}{ Test Teórico }} \\
\hline Grupo & TRAD & GIN & GIN & TRAD & & & & \\
\hline Clases (C) & $\begin{array}{l}\mathrm{C} 1 \\
\mathrm{C} 2\end{array}$ & $\mathrm{C} 4$ & $\begin{array}{l}\mathrm{C} 5 \\
\mathrm{C} 6\end{array}$ & $\mathrm{C} 3$ & $\begin{array}{ll}\mathrm{C} 1 \\
\mathrm{C} 2\end{array}$ & $\mathrm{C} 4$ & $\begin{array}{l}\mathrm{C} 5 \\
\mathrm{C} 6\end{array}$ & C3 \\
\hline
\end{tabular}

El objetivo de las sesiones estuvo enfocado a los contenidos para la ESO, correspondiente al bloque 2 de juegos y deportes. TRAD recibió los contenidos mediante una presentación de diapositivas realizada por un Graduado en Ciencias de la Actividad Física y el Deporte y estudiante del Máster de Educación en Profesorado de Educación Secundaria de la Universidad de Alicante. GIN se dividió en 5 grupos por los que el alumnado tenía 4 minutos para completar la actividad asociada a cada grupo rotativo. En los grupos interactivos es necesario un moderador encargado de asegurar que se cumpla el principio de diálogo igualitario como uno de los siete principios necesarios para el aprendizaje dialógico (Aubert, García, \& Racionero, 2014). En este caso, por cada grupo intervino un Graduado o Graduada en Ciencias de la Actividad Física y el Deporte y estudiante del Máster de Educación en Profesorado de Educación Secundaria de la Universidad de Alicante. Estos adquirían la figura de moderadores para los GIN como miembro de esta $\mathrm{CA}$. Al finalizar ambas intervenciones tanto en la clase TRAD como GIN, el alumnado contó con 15 minutos para completar cada una de las encuestas para medir la satisfacción de necesidades psicológicas básicas y motivación.

\section{Cuestionarios}

Escala de las Necesidades Psicológicas Básicas en el Ejercicio (BPNES) adaptada a la Educación Física (Moreno, González, Chillón, \& Parra, 2008).
Se utilizó la versión española de la Escala de Necesidades Psicológicas Básicas en el Ejercicio (BPNES) (Vlachopoulos \& Michailidou, 2006) adaptada al contexto de la Educación Física (Moreno, González, Chillón, \& Parra, 2008). Este cuestionario consta de 12 ítems agrupados en tres factores (cuatro ítems por cada factor) que miden la $\mathrm{A}, \mathrm{C}$ y RD, a los que se responde mediante una escala tipo Likert que abarca desde el número 1 (totalmente en desacuerdo) hasta el número 5 (totalmente de acuerdo). Los resultados revelaron que la escala era válida y fiable para medir la satisfacción de las tres necesidades psicológicas básicas en el ejercicio en las clases de EF con adolescentes españoles.

Regulación del Comportamiento en el Cuestionario de Ejercicio-2 (BREQ-2 (Moreno, Cervelló, \& Martínez, 2007).

La escala BREQ-2 tiene todas las condiciones requeridas en cuanto a los criterios de fiabilidad y validez para ser utilizados en el contexto español en diferentes áreas de la actividad física (Moreno, Cervelló, \& Martínez, 2007). Este cuestionario se compone de 19 ítems que miden regulación intrínseca, identificada, introyectada, externa y desmotivación. A los que se contesta mediante una escala tipo Likert abarcando desde el número 1 (totalmente en desacuerdo) hasta el número 5 (totalmente de acuerdo).

Test de conocimientos teóricos específicos de la asignatura.

El test fue llevado a cabo en un tiempo máximo de 20 minutos. Los contenidos teóricos que componían esta prueba de evaluación han sido extraídos a partir de los estándares de aprendizaje establecidos en la unidad didáctica correspondiente al momento del curso.

Para evaluar el conocimiento del alumnado en el deporte estudiado (fútbol), se utilizó un test específico, contabilizando la frecuencia de ítems correctos respecto a la historia, acciones técnicas y tácticas incluyendo las posiciones de los jugadores en el campo. El test quedó compuesto por 5 preguntas con valor de 2 puntos cada una, pudiendo obtener una máxima nota de 10 puntos. Todas las preguntas fueron corregidas a través de criterios objetivos desarrollados de la siguiente manera:

- Pregunta 1 (Historia del fútbol): 2 preguntas tipo test con 4 posibles respuestas puntuando con valor de un punto cada pregunta.

- Pregunta 2 (Técnica y táctica): 10 conceptos contando 0.2 puntos cada una de ellas únicamente como correcta $\mathrm{e}$ incorrecta a encuadrar como acciones técnicas o tácticas.

- Pregunta 3 (Acciones del juego): 2 acciones a elegir libremente entre 10 posibles puntuando 1 punto por cada definición correcta.

- Pregunta 4 (Sistemas de juego): Compuesta por dos conceptos, el primero sistema ofensivo y el segundo sistema defensivo, se valoraba con 0.5 puntos nombrar de forma numérica (por ejemplo: 4-4-2) un sistema de juego para cada concepto, y con otros 0.5 la justificación de por qué ese sistema correspondía al concepto adecuado. Sumando el punto referente al sistema de juego ofensivo y el punto del sistema de juego defensivo.

- Pregunta 5(Posiciones): Completar el espacio en blanco correspondiente a la posición correcta requerida en cada uno de los 4 ítems de los que constaba esta última pregunta. 


\section{Análisis estadístico de los datos}

Se utilizó el software Statistical Package for The Social Sciences (v.24.0 SPSS Inc., Chicago, IL, USA). La normalidad de la muestra fue comprobada mediante las pruebas K-S (Kolmogorov-Smirnov) y Shapiro-Wilk. Tras concluir que los datos no fueron paramétricos, se aplicó la prueba no paramétrica U de Mann - Whitney. El grado de significación fue establecido en $p<.05$.

\section{Resultados}

En primer lugar, se observaron diferencias estadísticamente significativas $(p=.018)$ entre los grupos TRAD y GIN para la variable Autonomía, siendo mayor en el grupo GIN tras la intervención (Tabla 2).

En segundo lugar, se observaron diferencias estadísticamente significativas en los dos niveles más altos de motivación, Regulación Intrínseca $(p=.002)$ y Regulación Identificada $(p=.016)$, siendo mayor en el grupo GIN (Tabla3).

Finalmente, también se pueden observar diferencias estadísticamente significativas ( $p<.001)$ favorables en GIN frente a TRAD (Tabla 4) en la evaluación del conjunto de conocimientos teóricos específicos de la asignatura.

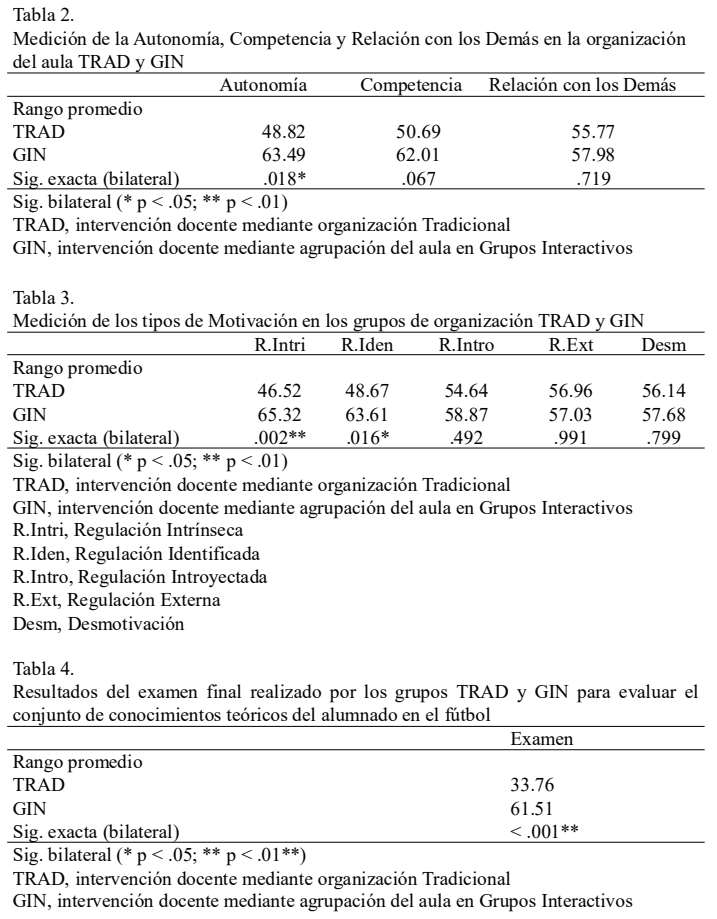

\section{Discusión, limitaciones y prospectiva}

Los resultados de este estudio mostraron que los grupos que recibieron las clases a través de agrupaciones basadas en GIN mostraron más Autonomía y niveles de motivación mas altos (Regulación Intrínseca e Identificada) y aumentaron la adquisición de contenidos teóricos respecto al grupo que recibió la clase mediante la organización de aula basada en TRAD.
Satisfacción de las Necesidades Psicológicas Básicas Primarias en el Ejercicio

En primer lugar, en relación con el análisis de las necesidades psicológicas básicas primarias en el ejercicio, únicamente se obtuvieron diferencias estadísticamente significativas, aceptando el planteamiento previo del grupo GIN en $\mathrm{A}$, sin obtenerse diferencias estadísticamente significativas en $C$ y RD. Estos resultados se relacionan con conclusiones como las de Moreno y González (2006) en las que se muestra que, la $\mathrm{A}$, junto a $\mathrm{C}$, son predictores de la motivación autodeterminada. En segundo lugar, referente a la $\mathrm{C}$, es importante destacar que, aunque no se hayan obtenido diferencias estadísticamente significativas, sí se aprecia una tendencia a favor de GIN (62.01) obteniendo una media superior al grupo TRAD (50.69). Con el objetivo de completar una de las teorías motivaciones de mayor relevancia en la actualidad, la Teoría de la Autodeterminación (Deci \& Ryan, 1980, 1985), se analizan también los ítems correspondientes a la medición de la RD. No se obtuvieron diferencias estadísticamente significativas en RD a favor de GIN frente a TRAD. Este bajo impacto de GIN en la RD podría estar vinculado al contexto de exclusión del alumnado, o simplemente a otras razones como una insuficiente explicación por parte del profesor al alumnado en la entrega del cuestionario. Esto justificaría que, unas actividades realizadas en grupo en las que el alumnado interactúa constantemente como las clases de GIN, puedan tener una insignificante diferencia en la media frente a las clases TRAD. En esta línea, cobrarían importancia afirmaciones como la de Ntoumanis (2001) que refleja como la $\mathrm{RD}$ expresada como necesidad de estar con los amigos o hacer nuevos amigos, es uno de los principales motivos de participación, lo que tendría que verse reflejado en los GIN. Aunque otros estudios como el de Amorose, Anderson-Butcher, Flesch, \& Klinefelter (2005), a través de un modelo de ecuaciones estructurales establecieron que la C y A percibida predecían la motivación autodeterminada, sin mostrar como predictor la RD.

Por último, se añade como prospectiva de estudio esta misma intervención, pero extrapolada al ámbito docente, contexto que, por su importancia, ya ha sido objetivo de estudio en intervenciones anteriores (Lieberman \& Miller, 2008; Quíles, Moreno, \& Lacárcel, 2015; Hunuk, Tannehill, \& Levent, 2019). De este modo, se propone el análisis de la relación del poder de soporte de autonomía, mediadores psicológicos y motivación autodeterminada con la satisfacción docente.

\section{Motivación}

Se observaron diferencias estadísticamente significativas en los ítems que miden la regulación del comportamiento en las clases TRAD y GIN referentes a la Regulación Intrínseca y Regulación Identificada, que son los niveles más altos de motivación.

Para explicar estos resultados es necesario entender la Teoría de la Autodeterminación señalada anteriormente. Según los autores Deci y Ryan (2000) las tres necesidades que componen esta teoría influirán en la motivación, de manera que el incremento de la percepción de A, C y RD creará un estado de motivación intrínseca, mientras que la frustración 
de éstas estará asociada con una menor motivación intrínseca y una mayor motivación extrínseca y desmotivación. Complementada a su vez con el Modelo Jerárquico de la Motivación Intrínseca y Extrínseca (Vallerand 1997, 2001) también explicado anteriormente. Silveira y Moreno (2015) analizaron los efectos de la manipulación del clima motivacional, y del feedback positivo tanto verbal como no verbal, relacionándolo tanto con la motivación intrínseca como con el miedo a equivocarse y la importancia que le concede el estudiante a la EF, estando esto mediado por la satisfacción de estas tres necesidades psicológicas básicas en el ejercicio. Concluyendo como una intervención efectiva ya que hubo mejoras en el grupo experimental en las variables motivacionales, viéndose disminuida la del miedo a fallar, apoyando esta relación entre necesidades psicológicas básicas en el ejercicio y motivación. Por su parte, en el ámbito deportivo, Moreno y González (2006) destacan como deportistas que percibían un estilo a favor de la autonomía y una cohesión de equipo, se sentían más competentes, autónomos y conectados con los demás, mostrando más motivación intrínseca y autodeterminada. Estos resultados obtenidos en deportistas que forman parte de un equipo podrían ser equiparables al ámbito educativo en alumnos que forman parte de un GIN durante la clase de EF. En la misma línea, Paava (2001), halló que la dimensión de intimar con los demás predecía positivamente la motivación intrínseca hacia la estimulación y la identificación. Del mismo modo, diferentes autores también han investigado las teorías motivacionales en el ámbito deportivo. En los que se refleja cómo la satisfacción de las tres necesidades psicológicas (A, C y RD) actuaron cómo predictor positivo de la motivación autodeterminada (Blanchard \& Vallerand, 1996). Estas investigaciones previas apoyan los resultados obtenidos en el presente estudio, en los que los GIN muestran tanto mayor A como mayores niveles de motivación.

\section{EF}

Adquisición de conocimientos teóricos específicos de

No se puede olvidar la importancia de la evaluación del conjunto de conocimientos que contribuyen al logro de los objetivos de cada enseñanza y etapa educativa y a la adquisición de competencias como recoge la LOMCE (Ley Orgánica 8/2013, sec. I, p. 9786). En relación con el grado de adquisición de conocimientos teóricos específicos de EF, los resultados muestran diferencias significativas a favor de GIN frente a TRAD aceptándose la hipótesis previa. Estos resultados son consistentes con estudios anteriores que compararon estilos cognitivos con tradicionales durante las clases prácticas de EF para evaluar el grado de adquisición conceptual de la nomenclatura, ubicación y aplicación de los músculos en el cuerpo humano (Muros et al., 2010). Por su parte, Chillón (2005) comprobó los efectos que tiene un programa de intervención de EF para la salud en adolescentes, obteniendo diferencias altamente significativas a favor del grupo que realizó la intervención con clases concretas de EF para la salud $(20.1 \pm 3.47)$ frente al que recibió las clases tradicionales $(13.34 \pm 28.32)$. Cabe destacar las similitudes en las características de las muestras utilizadas en los diferentes estudios empleados para la comparación, contraste y relación con la intervención realizada. Tales muestras, tanto la del presente estudio como la de los estudios contrastados, están compuestas en todos los casos por alumnos de la asignatura de EF pertenecientes a la etapa de la ESO.

\section{Conclusiones}

El objetivo del estudio fue analizar el tipo de agrupación en el aula mediante GIN para comprobar, por un lado, su impacto sobre la motivación y la satisfacción de las necesidades psicológicas básicas en el ejercicio, y por otro, en qué medida influyen estos GIN sobre el aprendizaje teórico. Los resultados mostraron que, en cuanto a la satisfacción de las necesidades psicológicas básicas en el ejercicio, únicamente puede aceptarse la hipótesis previa en el ítem correspondiente a la autonomía, sin poder hacerlo en la competencia y relación con los demás. Además, se obtuvieron diferencias significativas a favor de GIN en dos de las tres formas de motivación más autodeterminadas (regulación intrínseca e identificada) y en la evaluación del conjunto de conocimientos teóricos específicos de la asignatura. Estos hallazgos podrían ser útiles para futuros docentes que deberán entender el periodo de adolescencia en el que se encuentran sus alumnos, etapa en la que sería interesante no solo tener en cuenta la adquisición de conocimientos teóricos, sino también la satisfacción de las necesidades psicológicas básicas en el ejercicio como autonomía, competencia y relación con los demás, relacionadas con las diferentes formas de motivación del alumnado.

\section{Agradecimientos}

Agradecemos al centro educativo la implicación en esta investigación, que desde el participó activamente y facilitó todas las propuestas realizadas. Así como al alumnado universitario por su colaboración.

\section{Referencias}

Aubert, A., Flecha, A., García, C., Flecha, R., \& Racionero, S. (2008). Del enfoque individual al comunitario. El giro dialógico en las teorías del aprendizaje. Aprendizaje dialógico en la sociedad de la información, 27-90.

Aubert, A., Garcia, C., \& Racionero, S. (2009). El aprendizaje dialógico. Culturayeducación, 21(2), 129-139.

Amorose,A.,Anderson-Butcher, D., Flesch, S., \& Klinefelter, L. (2005). Perceived motivational climate and self-determined motivation in maleand female high school athletes. Research Quarterly for Exercise and Sport, 76.

Blanchard, C., \& Vallerand, R. J. (1996). Perceptions of competence, autonomy, and relatedness as psychological mediators of the social factors-contextual motivation relationship. Unpublished manuscript. Université du Québec à Montréal.

Bolam, R. (2000). Emerging policy trends: some implications for continuing professional development. Journal of In-Service Education, 26(2), 267-280.

Capllonch, M., \& Figueras, S. (2012). Educación Física y comunidades de aprendizaje. Estudios Pedagógicos, 38, 231-247.

Castro, M., Gómez,A., \& Macazaga,A. M. (2014). Aprendizaje dialógico y grupos interactivos en Educación Física. Retos, (25).

Chillón, P. (2005). Efectos de un programa de intervención de Educación Física para la salud en adolescentes de $3^{\circ}$ de ESO. Tesis doctoral inédita. Universidad de Granada 
Deci, E. L., \& Ryan, R. M. (1985). Intrinsic motivation and selfdetermination in human behavior. New York: Plenum.

Deci, E.L., \& Ryan, R. M.(1980). The empirical exploration of intrinsic motivational processes. En L. Berkowitz. Advances in Experimental Social Psychology, 13, 39-80. New York: Academic Press.

Deci. E. L., \& Ryan, R. M. (2000). The «what» and «why» of goal pursuits: Human needs and the self-determination of behaviour. Psychological Inquiry,11, 227-268.

Deglau, D., \& O'Sullivan, M. (2006). The effects of a long-term professional development program on the beliefs and practices of experienced teachers.

Desimone, L. M. (2011). A primer on effective professional development. Phi delta kappan, 92(6), 68-71.

Elboj, C., \& Niemelä, R. (2010). Sub-communities of mutual learners in the classroom: the case of Interactive groups. Revista de psicodidáctica, 15(2), 177-189.

Elboj, C., Puigdellívol, I., Soler, M., \& Valls, R. (2002). Comunidades de aprendizaje. Barcelona: Graó.

Escaravajal Rodríguez, J. C., \& Martín-Acosta, F. (2019). Análisis bibliográfico de la gamificación en Educación Física.

Fernández, M., \& Espadas, M. (2017). Formación inicial y percepción del profesorado sobre los estilos de enseñanza en Educación Física. Retos. Nuevas Tendencias en Educación Física, Deporte y Recreación,(31), 69-75.

Ferriz, A., Sebastiá, S., \& García, S. (2017). Clase invertida como elemento innovador en Educación Física: Efectos sobre la motivación y la adquisición de aprendizajes en Primaria y Bachillerato.

Flecha, R. (2001). Las comunidades de aprendizaje como expertas en resolución de conflictos. Ministerio de Educación, Cultura y Deporte (MEC).

García, I. G., Lemus, N. C., \& Morales, P. T. (2015). Las flipped classroom a través del smartphone: efectos de su experimentación en educación física secundaria. Prisma Social, (15), 296-352.

Gómez, A., Mello, R., Santa Cruz, I., \& Sordé, T. (2010). De las experiencias de Comunidades de Aprendizaje a las políticas basadas en sus éxitos. Revista Interuniversitaria de Formación del Profesorado, 67(24), 113-126.

Hortigüela-Alcalá, D., Fernández-Río, J., \& Pérez-Pueyo, A. (2016). Efectos del planteamiento docente en la enseñanza del futbol sobre el clima de aula. Percepciones de alumnadoy profesorado. Cuadernos de Psicología del Deporte, 16(1), 295-306.

Hunuk, D., Tannehill, D., \& Levent, M. (2019). Interaction patterns of physical education teachers in a professional learning community. Physical Education and Sport Pedagogy, 24(3), 301317.

Lieberman, A., \& Miller, L. (2008). Teachers in professional communities: Improving teaching and learning. Teachers College Press.

Moreno, J. A., González-Cutre, D., Chillón, M., \& Parra, N. (2008). Adaptación a la Educación Física de la escala de las necesidades psicológicas básicas en el ejercicio. Revista Mexicana de Psicología, 25(2), 295-303.

Moreno, J. A., \& González, D. (2006). El papel de la relación con los demás en la motivación deportiva. In VI Congreso Internacional de Educación Física e Interculturalidad. Murcia: ICD.

Moreno. J. A., Cervelló, E. C., \& Martínez, A. (2007). Measuring selfdetermination motivation in a physical fitness setting: validation of the Behavioral Regulation in Exercise Questionnaire-2 (BREQ-2) in a Spanish sample. Journal of Sports Medicine and Physical Fitness, 47(3), 366

Moreno, J. A., Zomeño, T. E., Marín, L. M., Cervello, E., \& Ruiz, L. M. (2009). Variables motivacionals relacionades amb la pràctica esportiva extraescolar en estudiants adolescents d'educació física. Apunts. Educació física i esports, 1(95), 38-43.

Muros, J. J., Som, A., Leyva, A. I., \& Zabala, M. (2010). Efecto de dos estilos de enseñanza (cognoscitivo versus tradicional) sobre el apren- dizaje de conceptos de anatomía muscular en alumnos de Educación Física de $1^{\circ}$ de ESO. Apunts. Educació física i esports, 100 (23).

Ntoumanis, N. (2001). A self-determination approach to the understanding of motivation in physical education. British Journal of Educational Psychology, $\quad 71,225-242$.

Paava, M. (2001). Motivation and perceived relatedness. Chicago.

Patton, K., Parker, M., \& Pratt, E. (2013). Meaningful learning in professional development: Teaching without telling. Journal of Teaching in Physical Education, 32(4), 441-459.

Pelletier, L., Fortier, M., Vallerand, R., Tuson, K., Briere, N., \& Blais, M. (1995). Toward a new measure of intrinsic motivation, extrinsic motivation, andamotivation in sports: The Sport Motivation Scale (SMS). Journal of Sport and Exercise Psychology, 17, 35-53.

Pérez-Pueyo, Á., Martínez-Samperio, C., \& Garrote-García., J. (2008). Metodología: un término confuso que todos parecemos tener claro. In IVCongreso Internacional y XXV Nacional de Educación Física (Córdoba, 2-5 de abril de 2008):» los hombres enseñando aprenden». Séneca (Epst. 7, 8) (p. 345).

Quíles, M. R., Moreno, J. A., \& Lacárcel, J. A. (2015). Del soporte de autonomía y la motivación autodeterminada a la satisfacción docente. European Journal of Education and Psychology, 8(2), 6875.

Racionero, S., \& Padrós, M. (2010). The dialogic turn in educational psychology. Revista de Psicodidáctica, 15(2), 143-162.

Ruiz, L. M., Moreno, J.A., Ramón, I., \& Alias, A. (2015). Motivación de Logro para Aprender en Educación Física: adaptación de la versión española del Test AMPET. Revista española de pedagogía, 157-175.

Ryan, R. M., \& Deci, E. L. (2000). Self-determination theory and the facilitation of intrinsic motivation, social development and wellbeing. American Psychologist, 55, 68-78.

Silveira, Y., \& Moreno, J.A. (2015). Miedo a equivocarse y motivación autodeterminada en estudiantes adolescentes. Cuadernos de Psicología del Deporte, 15(3), 65-74.

Vallerand, R. J. (1997). Toward a hierarchical model of intrinsic and extrinsic motivation. Advances in experimental social psychology, 29, 271-360.

Vallerand, R. J. (2001). A hierarchical model of intrinsic and extrinsic motivation in sport and exercise. En G.C. Roberts (Ed.), Advances in motivation in sport and exercise (pp. 263-319). Champaign, IL: Human Kinetics.

Vallerand, R. J. (2007). A hierarchical model of intrinsic and extrinsic motivation for sport and physical activity. Advances in motivation in sport and exercise, 263-319.

Valls, R., \& Kyriakides, L. (2013). The power of Interactive Groups: how diversity of adults volunteering in classroom groups can promote inclusion and success for children of vulnerable minority ethnic populations. Cambridge journal of education, 43(1), 17-33.

Vlachopoulos, S. P., \& Michailidou, S. (2006). Development and initial validation of a measure of autonomy, competence, and relatedness in exercise: The Basic Psychological Needs in Exercise Scale. Measurement in physical education and exercise science, 10(3), 179-201.

Wenger, E. 2013. Communities of Practice: ABrief Introduction. Accedido el 10 de Febrero, 2019. http://wenger-trayner.com/wp-content/ uploads/2013/10/06-Brief-introduction-to-communities-ofpractice.pdf.

Zhang, H. (2016). Research on innovation of physical education teaching method based on inquiry teaching: A survey based on online questionnaire. International Journal of Future Generation Communication and Networking, 9(2), 37-48. 